\title{
Molecular Mobility of Polyepoxide Networks As Revealed by Emission Spectroscopy and Mechanical Analysis
}

\author{
E. W. Meijer* and R. J. M. Zwiers \\ Philips Research Laboratories, 5600 JA Eindhoven, The Netherlands. Received May 7, 1986
}

\begin{abstract}
This paper presents the use of phosphorescence spectroscopy to reveal the molecular mobility of polyepoxide networks. The polyepoxides are prepared by a photoinitiated cationic polymerization and cross-linking of (cyclo)aliphatic bisepoxides. These networks emit fluorescence $\left(\lambda_{\max }=340 \mathrm{~nm}\right)$ and phosphorescence $\left(\lambda_{\max }=480 \mathrm{~nm}\right)$ originating from intermediates in the photolysis of the initiator bis(4-tert-butylphenyl)iodonium hexafluoroarsenate. The emission properties have been studied as a function of temperature. Both phosphorescence intensity and triplet excited state half-life show a discontinuity in their Arrhenius plots. For all networks this discontinuity is situated around $260 \mathrm{~K}$ and is due to the onset of quenching of the triplet excited state. The activation energy above the transition point is characteristic of the individual networks and is an indication of the increase in motion as the temperature is raised, ultimately leading to a simultaneous motion of large parts of the network. From these emission data the glass transition temperature and the width of the glass transition region have been estimated. The mechanical properties of the network are established with dynamic mechanical thermal analysis (DMTA) measurements. A striking correlation between molecular mobility (from emission data) and mechanical properties (from DMTA) is observed.
\end{abstract}

\section{Introduction}

The macroscopic mechanical properties of polymer networks depend on the characteristics of the network structure and its molecular mobility. The complicated nature of densely cross-linked polymers has limited the investigations to the structural dynamics of these materials, possibly due to the relatively limited number of suitable experimental techniques. ${ }^{1}$ In the case of highly crosslinked polyepoxide networks, the studies that have been reported are related merely to epoxide-amine networks, using, for instance, torsional braid analysis or dynamic mechanical thermal analysis, ${ }^{2-5}$ solid-state NMR spectroscopy, ${ }^{6}$ and ESR spectroscopy. ${ }^{7}$ At present there is considerable interest in the photoinitiated cationic homopolymerization of epoxides. ${ }^{8-11}$ However, information about the structural dynamics of the polymer networks produced is scarce.

In this study we have used emission spectroscopy to obtain information about the network microstructure of these polyepoxides. In recent years the application of emission spectroscopy to the study of a variety of phenomena in linear polymers has become widespread. ${ }^{12}$ The luminescence of a chromophore incorporated in a polymer is in many cases very sensitive to properties of the local environment.

Important emission parameters are intensity, lifetime data, and degree of depolarization of both fluorescence and phosphorescence. Using the appropriate luminescent probe and monitoring the parameters to match, one can sensitively measure properties like polymer compatibility, ordering of polymer chains, viscosity, glass transition temperatures, micromobility, subgroup motion, or micropolarity..$^{12-18}$

The large difference in rate for the $\mathrm{S} 1 \rightarrow \mathrm{S} 0$ spin-allowed transition $\left(10^{6} / 10^{9} \mathrm{~s}^{-1}\right)$ and the $\mathrm{T} 1 \rightarrow \mathrm{S} 0$ spin-forbidden transition $\left(10^{-1} / 10^{2} \mathrm{~s}^{-1}\right)$ opens the possibility of studying molecular motion at a great diversity of frequencies. ${ }^{12,19}$

Recently we observed remarkable luminescent properties in the polymer matrix when aliphatic and cycloaliphatic bisepoxides were polymerized with diaryliodonium salts and UV light. ${ }^{20}$ Both fluorescence and phosphorescence were obtained at $\lambda_{\max }=340$ and $480 \mathrm{~nm}$, respectively. The latter showed a nearly exponential decay with a half-life of $1.6 \mathrm{~s}$ at ambient temperature. The ratio $I_{\mathrm{p}} / I_{\mathrm{f}}$ as a function of the degree of polymerization depended on the structure and reactivity of the monomers used, yielding a new technique to determine the stage of polymerization
Chart I<smiles>C(CCC1CO1)COCC1CO1</smiles><smiles>C1CCC2OC2C1</smiles>

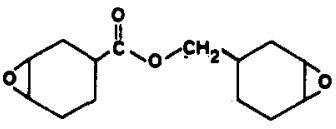

III

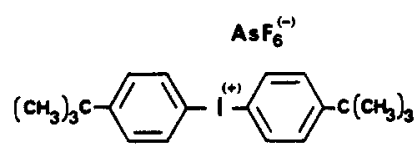

and cross-linking. ${ }^{20}$ The lifetime of the triplet excited state appeared to be constant during conversion, which prompted us to conclude that cationic photopolymerization of bisepoxides occurs via an inhomogeneous polymerization process. ${ }^{20} \mathrm{~A}$ few studies have recently been presented for monitoring polymerization and cross-linking in epoxyamine networks by fluorescence techniques ${ }^{21,22}$ and in polyacrylate networks by depolarization in fluorescence. ${ }^{23}$

Hitherto no investigations using emission spectroscopy in order to study the structure and structural dynamics of polymer networks as a function of temperature have been presented. In this paper we describe the results of our study to the molecular mobility of (cyclo)aliphatic polyepoxide networks at complete conversion as a function of temperature. These results are compared with mechanical data obtained by dynamical mechanical thermal analysis (DMTA).

\section{Experimental Section}

The bisepoxides studied comprise 1-(epoxyethyl)-3,4-epoxycyclohexane (I) (Union Carbide), 1,4-butanediol diglycidyl ether (II) (Ciba Geigy), and (3,4-epoxycyclohexyl)methyl $3^{\prime}, 4^{\prime}$-epoxycyclohexanecarboxylate (III) (Ciba Geigy) (see Chart I). 1,2Epoxycyclohexane (IV) (Janssen Chemicals) was used as monoepoxide. The bisepoxide monomers were all used as purchased. The purity of the samples proved to be $\left({ }^{1} \mathrm{H}\right.$ NMR $)$ spectroscopically pure for I, II, and IV. For one set of experiments bisepoxide III was purified by column chromatography $\left(\mathrm{Al}_{2} \mathrm{O}_{3}-\mathrm{CH}_{2} \mathrm{Cl}_{2}\right)$ in order to remove polar impurities. No serious attempts have been made to remove the small amount of water that is possibly present in the samples. The photoinitiator, bis(4-tert-butylphenyl)iodonium hexafluoroarsenate $(\mathrm{V})$, was synthesized by a well-known procedure ${ }^{8}$ with some minor modifications.

The emission spectra of the networks were measured from freshly prepared films of the monomers on optical grade aluminum. In all cases the amount of photoinitiator in the bisepoxides was $2 \mathrm{~mol} \%$. The specimens were polymerized by irra- 


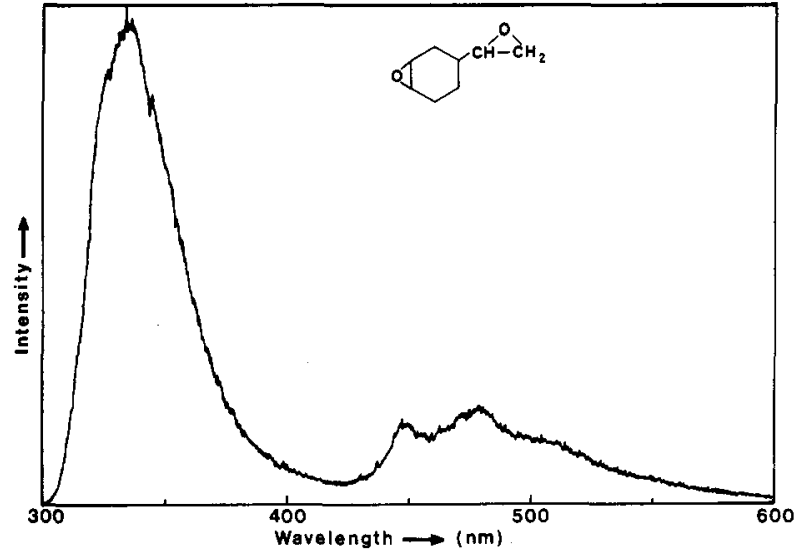

Figure 1. Emission spectrum of a network of 1-(epoxyethyl)3,4-epoxycyclohexane (I) upon excitation at $\lambda=254 \mathrm{~nm}$.

diation at $\lambda=254 \mathrm{~nm}$ at ambient temperature by use of a Camag lamp with an intensity of about $1 \mathrm{~mW} / \mathrm{cm}^{2}$. In order to determine the irradiation time necessary for maximum conversion at ambient temperature, a specially equipped Perkin-Elmer DSC 2 apparatus was used.

The emission spectra were recorded on a Monospek 1000 spectrophotometer with a focal length of $100 \mathrm{~cm}$. The spectrophotometer was equipped with a 1-m monochromator and a cooled photomultiplier with an RCA 031034 tube, and photocounting was used. A high-pressure mercury lamp (Philips HPK $75 \mathrm{~W}$ ) was used as the light source. The excitation was filtered by two 254-nm interference filters, and the emission was filtered by a (WG5) cutoff filter. A Canberra S30 multichannel analyzer interfaced with a microcomputer was used for data acquisition. Decay measurements were performed on the same instrument at the emission wavelength of $\lambda=480 \mathrm{~nm}$. To accumulate scans, an electronically driven shutter, controlled by the multichannel analyzer, was used. Excitation spectra were recorded on a Spex 1680 spectramate spectrophotometer with a double monochromator and a focal length of $22 \mathrm{~cm}$ using the same equipment as mentioned above for data aquisition.

DMTA measurements were performed with a Polymer Labs apparatus. Specimens with typical dimensions of $60 \times 10 \times 1$ $\mathrm{mm}$ were prepared by means of the Camag lamp and measured in the dual-cantilever mode. Moduli and $\tan \delta$ were determined in one scan from -100 to $200^{\circ} \mathrm{C}$ with a scan rate of $2{ }^{\circ} \mathrm{C} / \mathrm{min}$ at alternating frequencies of $0.1,1$, and $10 \mathrm{~Hz}$.

\section{Results}

The polyepoxide networks were obtained by a cationic polymerization at room temperature. Brønsted acid was generated photochemically by the irradiation of diaryliodonium salts with UV light at $254 \mathrm{~nm} .^{8-10}$ Intermediates in the photolysis of the iodonium salt are responsible for the observed emission behavior. ${ }^{20}$

Figure 1 gives a typical plot showing the emission spectrum of a polyepoxide network of 1-(epoxyethyl)-3,4epoxycyclohexane (I) recorded at room temperature without the exclusion of oxygen. The fluorescence around $\lambda=340 \mathrm{~nm}$ is accompanied by phosphorescence around $\lambda=480 \mathrm{~nm}$. The latter shows some vibrational fine structure. With decreasing temperature the molecular rigidity of the network is increased, furnishing a decrease in quenching rate of the triplet excited state, and hence an increase in phosphorescence intensity is expected. Several polyepoxide networks were investigated as a function of temperature. In Figure 2 the effect of temperature on the luminescence intensity of the polymer network of 1,4-butanediol diglycidyl ether (II) is given when it is irradiated with light of $254 \mathrm{~nm}$. The fluorescence intensity is increased by a factor of 1.3 going from $T=269$ to $193 \mathrm{~K}$. The change in phosphorescence intensity is dramatic: no phosphorescence at room temperature and

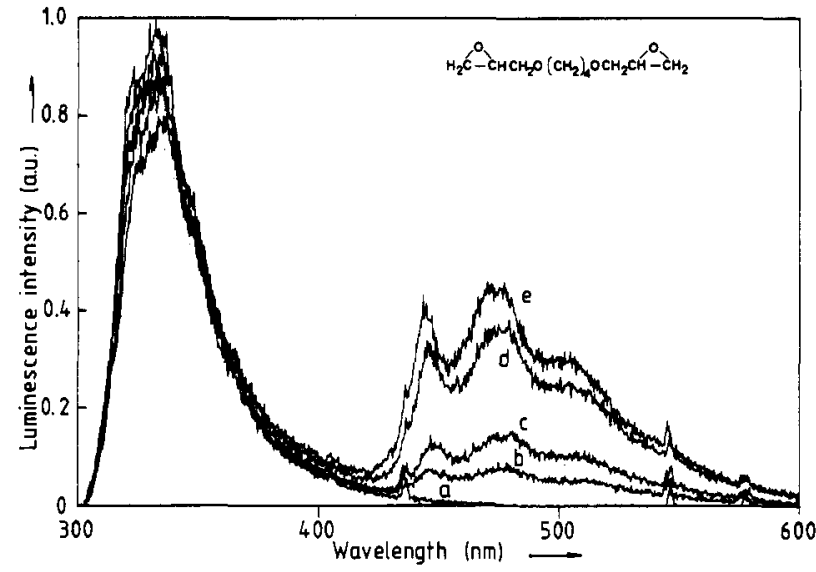

Figure 2. Emission behavior (excitation wavelength $254 \mathrm{~nm}$ ) of a network of 1,4-butanediol diglycidyl ether (II) as a function of temperature: (a) $296 \mathrm{~K}$; (b) $279 \mathrm{~K}$; (c) $273 \mathrm{~K}$; (d) $233 \mathrm{~K}$; (e) 193 $\mathrm{K}$.

a phosphorescence intensity almost half the fluorescence intensity at $193 \mathrm{~K}$. The half-life of the triplet excited state was determined at all temperatures at $480 \mathrm{~nm}$ (the wavelength at the maximum phosphorescence intensity without the contribution of the shoulder of the fluorescence). At all temperatures the decay curve proves to be nearly single exponential.

Since no marked differences in the mechanisms of absorption, intersystem crossing, and emission are to be expected between linear polymers and polymer networks, we use in this study the assumptions that are generally accepted for linear polymers. ${ }^{12}$ The following expression for the phosphorescence intensity $I_{\mathrm{p}}$ is well established: ${ }^{13}$

$$
I_{\mathrm{p}}^{-1}-I_{\mathrm{p} 0}{ }^{-1}=A \exp \left(-E_{\mathrm{a}}{ }^{\prime} / R T\right)
$$

When the fluorescence intensity is also considered, relationship 2 seems to be more appropriate ${ }^{14}$

$$
\left(I_{\mathrm{f}} / I_{\mathrm{p}}\right)\left(I_{\mathrm{p} 0} / I_{\mathrm{f} 0}\right)-1=B \exp \left(-E_{\mathrm{a}}{ }^{\prime \prime} / R T\right)
$$

where $I_{\mathrm{f}}, I_{\mathrm{p}}, I_{\mathrm{f} 0}$, and $I_{\mathrm{p} 0}$ are the fluorescence and phosphoresence intensities at temperature $T$ and the low-temperature limit, respectively, $A$ and $B$ are constants, and $E_{\mathrm{a}}$ is the energy term governing the temperature dependence of the phosphorescence. We have used in this study relationship 2, in which $I_{\mathrm{f} 0}$ and $I_{\mathrm{p} 0}$ are the intensities at $T=100 \mathrm{~K}$ of fluorescence and phosphorescence, respectively. The observed half-life of the triplet excited state is related to the rate constants of radiative $\left(k_{\mathrm{r}}\right)$ and nonradiative $\left(k_{\mathrm{nr}}\right)$ decay:

$$
\tau^{-1}=k_{\mathrm{r}}+k_{\mathrm{nr}}
$$

As for the phosphorescence intensity, a similar Arrhenius expression holds

$$
\tau^{-1}-\tau_{0}^{-1}=C \exp \left(-E_{\mathrm{a}}^{\prime \prime \prime} / R T\right)
$$

where $\tau_{0}{ }^{-1}$ is the low-temperature limit of the half-life (in our study it is the estimated half-life at $T=100 \mathrm{~K}$ ). Thus, Arrhenius plots of $\ln \left(\left(I_{\mathrm{f}} / I_{\mathrm{p}}\right)\left(I_{\mathrm{p} 0} / I_{\mathrm{fo}}\right)-1\right)$ and $\ln \left(\tau^{-1}-\tau_{0}^{-1}\right)$ vs. $1 / T$ should give straight lines of slope $\left(-E_{\mathrm{a}} / R\right)$. Figure 3 shows such Arrhenius plots for 1,4-butanediol diglycidyl ether (II). Both curves show distinct linear parts with a discontinuity in the slope within a narrow temperature region centered at $T=260 \mathrm{~K}$. The estimated activation energies from the intensity data are $E_{\mathrm{a}}=9 \mathrm{~kJ} / \mathrm{mol}$ in the low-temperature region and $E_{\mathrm{a}}>150 \mathrm{~kJ} / \mathrm{mol}$ above the transition temperature.

In Figure 4 the data are given for a network prepared from 1-(epoxyethyl)-3,4-epoxycyclohexane (I). It repre- 


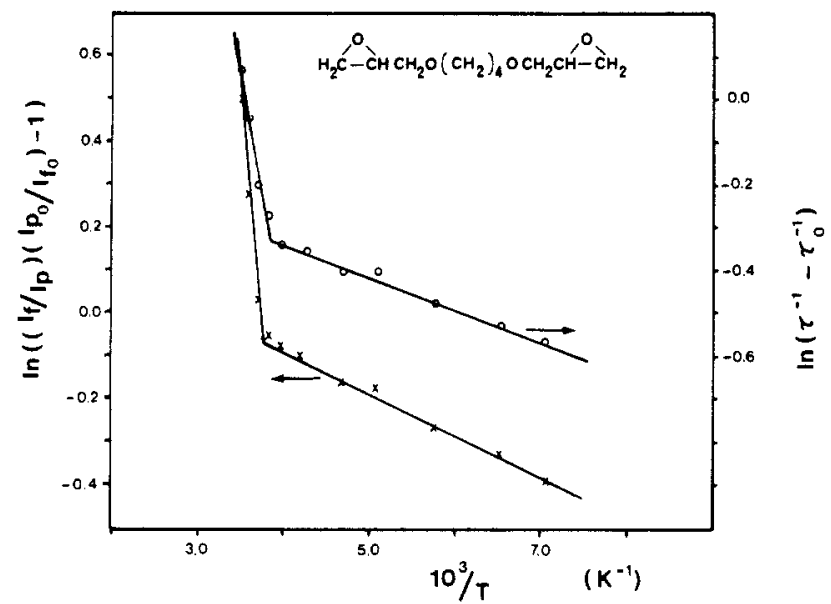

Figure 3. Arrhenius plot showing the temperature dependence of the phosphorescence intensity and the triplet excited state half-life of the network of 1,4-butanediol diglycidyl ether (II).

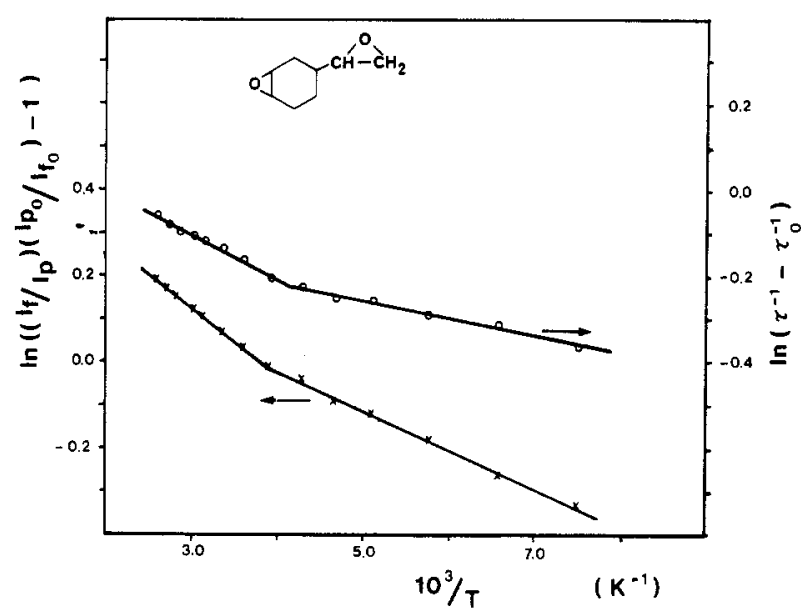

Figure 4. Arrhenius plot showing the temperature dependence of the phosphorescence intensity and the triplet excited state half-life of the network of 1-(epoxyethyl)-3,4-epoxycyclohexane (I).

sents an example in which there is little difference in activation energy above and below the transition point. This small difference hampers an accurate determination of the transition temperature.

The other individual bisepoxides and mixtures thereof show a temperature-dependent behavior comparable to that of the networks from I and II. The data are listed in Table I for both intensity and half-life. The activation energies below the transition point are very low and almost independent of the molecular structure of the monomer and hence of the polyepoxide network. These $E_{\mathrm{a}}$ values are similar to those found for linear polymers in the very low temperature region. ${ }^{12,13,15}$ Above the transition temperature the activation energies are very sensitive to the microstructure of the polyepoxides.

The transition points of all polyepoxide networks studied are situated around $260 \mathrm{~K}$. As can be seen from Table I, the activation energies as estimated from the intensity data are always higher than those estimated from the triplet excited state half-life data.

The temperature dependence of the emission from the polyepoxides is influenced by the thermal history of the sample. There is a clear difference in the phosphorescence intensity between measurements recorded as the sample was cooled by $20 \mathrm{~K}$ intervals compared to results obtained if the temperature of the sample is raised by $20 \mathrm{~K}$ intervals after slow cooling to $130 \mathrm{~K}$ (see Figure 5). The intensity
Table I

\begin{tabular}{|c|c|c|c|c|c|c|}
\hline \multirow[b]{2}{*}{ polymer network } & \multicolumn{3}{|c|}{ intensity } & \multicolumn{3}{|c|}{ half-life } \\
\hline & $\overline{E_{\mathrm{a} 1}{ }^{a}}$ & $T^{b}$ & $\overline{E_{\mathrm{n} 2}{ }^{\mathrm{C}}}$ & $\overline{E_{\mathrm{a} 1}{ }^{\circ}}$ & $T^{b}$ & $\overline{E_{\mathrm{a} 2} \mathrm{c}}$ \\
\hline I & 8 & 259 & 14 & 3 & $240-250$ & 9 \\
\hline II & 8 & 262 & 170 & 6 & 263 & 115 \\
\hline I:II $(4: 6 \mathrm{w}$ & 8 & 266 & 22 & 5 & 263 & 17 \\
\hline III & 6 & $225-257$ & 23 & 5 & $245-250$ & 10 \\
\hline III:IV (5:5 w/w) & 4 & 259 & 42 & 3 & 259 & 14 \\
\hline
\end{tabular}

${ }^{a}$ Activation energy in $\mathrm{kJ} / \mathrm{mol}$ as determined at the temperature region below the transition temperature $T$. ${ }^{b}$ Transition temperature in $\mathrm{K}$. ${ }^{\mathrm{c}}$ Activation energy in $\mathrm{kJ} / \mathrm{mol}$ as determined at the temperature region above the transition temperature $T$.

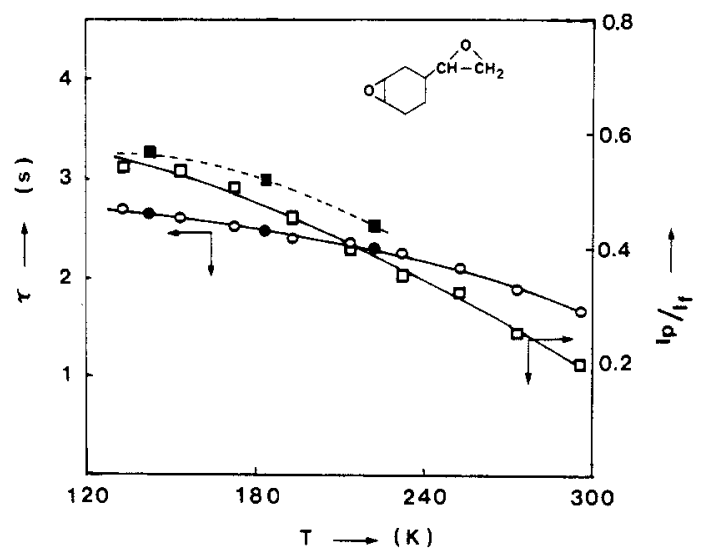

Figure 5. Effect of temperature history on the emission behavior of a network of I. Open circles and squares reflect decreasing temperature, and closed circles and squares reflect increasing temperature.

of the phosphorescence is higher in the case of measurements with increasing temperature than in the case of measurements with decreasing temperature. In contrast with the intensity data no history effect is found for the observed half-life of the triplet excited state.

In order to relate the local molecular mobility, as obtained from the emission measurements, with mechanical properties of the individual polyepoxide networks, we performed dynamic mechanical thermal analysis on these networks. The DMTA measurements were performed at the frequencies of 10,1 , and $0.1 \mathrm{~Hz}$. The $\tan \delta \mathrm{vs}$. temperature data for the individual bisepoxides and mixtures thereof are given in Figure 6. In the upper part of Figure 6 the networks of 1-(epoxyethyl)-1,2-epoxycyclohexane (I), 1,4-butanediol diglycidyl ether (II), and a mixture of I and II $(4: 6 \mathrm{w} / \mathrm{w})$ are compared. Network I shows a transition in a broad temperature range centered at $T=400 \mathrm{~K}$, while the main transition of II occurs in a short temperature region centered at $T=310 \mathrm{~K}$. The mixture shows a somewhat intermediate behavior; the main transition is a combination of two transitions. All three networks exhibit a low-temperature transition at almost the same temperature around $T=213 \mathrm{~K}$. The lower part of Figure 6 shows the results of two samples of $(3,4$-epoxycyclohexyl)methyl $3^{\prime}, 4^{\prime}$-epoxycyclohexanecarboxylate (III). The data with the broad transition centered at $T=338 \mathrm{~K}$ correspond to a network prepared from a technical batch of III, while the other results with the main transition centered at $T=428 \mathrm{~K}$ correspond to the network prepared from a sample of III in which the polar impurities present in the technical batch were removed with column chromatography.

Similar to the other polyepoxides, both samples of III exhibit the low-temperature transition at $T=220 \mathrm{~K}$.

In order to compare the mechanical properties with the micromobility of the polymer networks, we determined the 


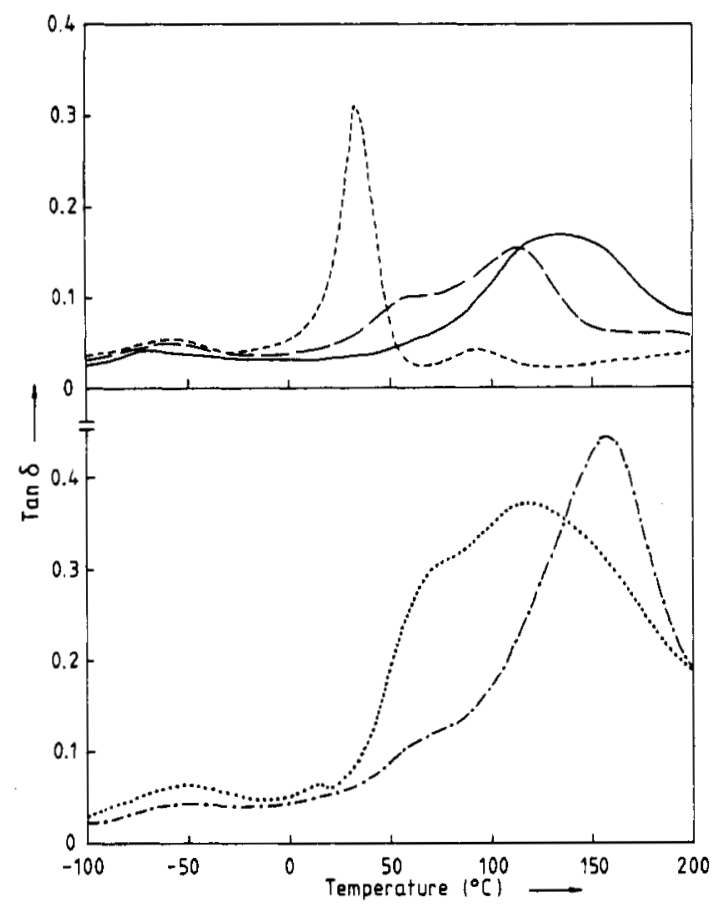

Figure 6. Tan $\delta$ as a function of temperature for the polyepoxide networks, measured at a frequency of $1 \mathrm{~Hz}$ : (-) I; (---) II; (---) mixture of I and II $(4: 6 \mathrm{w} / \mathrm{w}) ;(\cdot .$.$) III, technical batch; (-\cdot-)$ III, purified material.

Table II ${ }^{a}$

\begin{tabular}{lccl}
\hline $\begin{array}{c}\text { polymer } \\
\text { network }\end{array}$ & $\Delta \log E^{\prime}, \mathrm{Pa}$ & $\begin{array}{c}\Delta \log E^{\prime} / K, \\
\mathrm{mPa} / \mathrm{K}(T, \mathrm{~K})\end{array}$ & trans temp, ${ }^{b} \mathrm{~K}$ \\
\hline I & 0.4 & $6(400)$ & $210 ; 400^{*}$ \\
II & 1.2 & $47(310)$ & $215 ; 310^{*} ; 370$ \\
I:II $(4: 6 \mathrm{ww})$ & 0.6 & $12(390)$ & $215 ; 325^{*} ; 390^{*}$ \\
III & 0.55 & $12.5(325)$ & $220 ; 325^{*} ; 390^{*}$ \\
& 0.8 & $12.5(390)$ & \\
III (purified) & 0.6 & $17(425)$ & $220 ; 330 ; 425^{*}$
\end{tabular}

${ }^{a}$ Dynamic mechanical thermal analysis at $1 \mathrm{~Hz} .{ }^{b}$ Main transitions are noted with an asterisk.

decrease in dynamic modulus per degree $\left(\Delta \log E^{\prime} / K\right)$ at the main transition. These results, together with the total decrease in dynamic modulus ( $\Delta \log E$ ) at the main transition and the temperatures of the other transitions, are tabulated in Table II. The values of $\Delta \log E^{\prime} / K$ and $\Delta \log E^{\prime}$ at the main transition correspond to the width of that main transition, indicating the increase of polymer mobility per degree.

\section{Discussion}

The observations presented in this paper demonstrate the discontinuous behavior of phosphorescence as a function of temperature in highly cross-linked polyepoxide networks. The sensitivity of the lifetime of a triplet excited state in a host polymer matrix is determined by the microenvironment presented by the matrix and is expressed by equation 3 .

The conglomerate term $k_{\mathrm{nr}}$ is the rate sum of all the physical and chemical radiationless processes taking place from the lowest triplet state. These radiationless processes are influenced by the temperature, resulting in a decrease in rate as the polymer matrix is cooled. Hence, the observed lifetime of the triplet excited state is increased.

Guillet et al. have discussed the three possible components for $k_{n n}$, the rates of intramolecular decay, intermolecular (collisional) deactivation, and a bimolecular quenching process, in their paper concerning the photo- chemistry of ketone polymers. ${ }^{12}$ They attribute the discontinuous decrease in phosphorescence of polymers with rising temperature to the increase in the efficiency of oxygen quenching of the triplet excited state. Moreover, the motion of molecular oxygen in a polymer matrix is directly associated with the micro Brownian motion of small groups in the polymer. ${ }^{12,13}$ In our investigations toward the structure of polyepoxide networks we are prompted to conclude that the conclusions as drawn by Guillet for linear polymers hold for the polymer networks discussed in this paper as well, despite the fact that in our case the exact nature of the emitting species is still unknown. We will discuss our results in general terms of subgroup motion, resulting in quenching of the triplet excited state in the polymer network, without going into details of the mechanisms of these radiationless deactivations.

In the densely cross-linked polyepoxide networks studied so far, the transition point in the Arrhenius plots of phosphorescence intensity and half-life of the triplet excited state is always situated around the temperature 260 $\mathrm{K}$ and is hardly dependent on the structure of the network. This indicates that the onset of quenching of the emitting species in its triplet excited state is due to the onset of motion of a subgroup that all networks have in common. Since all materials consist of cross-linked (cyclo)aliphatic bisepoxides, it is reasonable to assume that the onset of bond rotation in the ethylene oxide functional group of the backbone is responsible for this quenching.

Below the transition temperature the radiationless deactivations are more or less excluded. The observed activation energies for both intensity and half-life data are very low $(<10 \mathrm{~kJ} / \mathrm{mol})$ and hardly dependent on the molecular structure of the monomer and hence of the polyepoxide network. These $E_{\mathrm{a}}$ values are comparable with those found for linear polymers in the very low temperature region. ${ }^{12,13,15}$ This indicates that, with respect to the frequency scale, the difference in molecular motion of polymers with physical cross-links (entanglements) and of polymers with chemical cross-links is only marginal in the glassy state (far) below $T_{\mathrm{g}}$.

Above the transition temperature, around $260 \mathrm{~K}$, the differences in activation energies for the individual epoxides are substantial. In this temperature region the structural differences in the bisepoxide monomers are expressed in the $E_{\mathrm{a}}$ values. The individual $E_{\mathrm{a}}$ values correspond to the increase in quenching of the excited emitting species in each network as the temperature is raised.

In polyepoxide I the increase in molecular mobility by raising the temperature is very small, indicating the rigidity of the network above $260 \mathrm{~K}$. In other words, the onset of subgroup motion of the substituted poly(ethylene oxide) backbone is succeeded by only a minor increase of other subgroup motions as the temperature is raised. Therefore a simultaneous motion of large parts of polymer network I is only expected at high temperatures. Responsible for the low activation energy are probably the rigidity of the cycloaliphatic group between the two epoxide functional groups in I, the variety of structures for the cross-links due to the difference in reactivity of the epoxides, and an inhomogenous polymerization process. ${ }^{20,24}$

In sharp contrast with the data of I are the results for network II prepared from 1,4-butanediol diglycidyl ether (II). In this case the increase in molecular mobility is considerable when the temperature is raised above the transition temperature at $262 \mathrm{~K}$. The very flexible $\mathrm{CH}_{2-}$ $\mathrm{OCH}_{2} \mathrm{CH}_{2} \mathrm{CH}_{2} \mathrm{CH}_{2} \mathrm{OCH}_{2}$ unit connecting the two epoxide groups in II causes a fast increase in quenching of the 


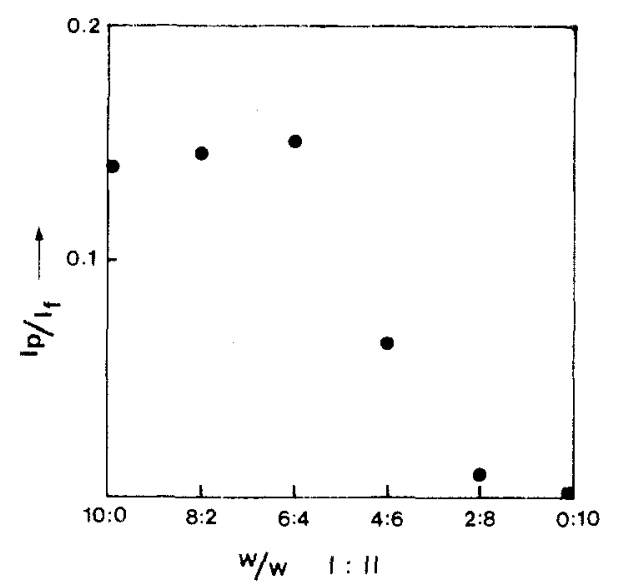

Figure 7. Phosphorescence intensity at maximum conversion at ambient temperature as a function of composition for networks of $I$ and II and mixtures thereof.

triplet excited state at relatively low temperatures, resulting in an activation energy well above $100 \mathrm{~kJ} / \mathrm{mol}$. From this high $E_{\mathrm{a}}$ value we expect a low glass transition temperature as well as a short temperature range in which this transition takes place.

Although the temperature behavior of the mixture of bisepoxides I and II (4:6 w/w) is somewhere between the characteristics of the individual monomers, the activation energy above the transition at $266 \mathrm{~K}$ is not the weighted average as could be expected on the basis of the composition of the mixture. The observed activation energy of $22 \mathrm{~kJ} / \mathrm{mol}$ for the intensity data indicates that the molecular mobility of the network resembles more that of polyepoxide I ( $14 \mathrm{~kJ} / \mathrm{mol}$ ) than that of polyepoxide II (170 $\mathrm{kJ} / \mathrm{mol}$ ), despite the excess of monomer II over I in the mixture. Since the decay of the triplet excited state proves to be single exponential at all temperatures, it is reasonable to exclude a macroscopic two-phase polymer network. This statement is in agreement with the observation that the rate of network formation of I is gradually decreased upon addition of bisepoxide II, as previously reported. ${ }^{20}$ Moreover, the networks obtained are completely transparent. The intensity of the phosphorescence of several mixtures of I and II at maximum conversion at ambient temperature is presented in Figure 7. DSC experiments reveal that the overall epoxy group conversion is slightly increased in the series as II is added to I. These data suggest that a network produced from I becomes more rigid on the molecular level upon addition of small amounts of II, while large amounts of II in the mixture produce a network in which the rigidity of $I$ is overshadowed by the flexibility of II. It is reasonable to assume that a high conversion of I at room temperature is hampered by steric hindrance and chain immobility due to the rigidity of $\mathrm{I}$. The addition of small amounts of II will increase the maximum conversion of $I$, because the flexible bisepoxide II can bridge the cap between two epoxide groups of I. Hence the addition of II results in a higher overall crosslink density and a more rigid network, despite the plasticizing role of bisepoxide II. At high concentrations of II in the mixture the increase in cross-link density is small and a rapid increase in mobility occurs upon addition of more II.

The molecular mobility and subgroup motion of the network obtained from (3,4-epoxycyclohexyl)methyl $3^{\prime}, 4^{\prime}$-epoxycyclohexanecarboxylate (III) are identical, within experimental error, with that of the 4:6 w/w mixture of I and II when these parameters are based on the emission spectra. Recent experiments show that technical batches of III are contaminated with small amounts of polar monofunctional epoxides. Therefore, it is expected that a network of pure III will exhibit a lower activation energy than the samples used in this emission study. The mechanical analysis, discussed below, confirms this statement. The addition of cyclohexene oxide (IV) to III leads to a polyepoxide network with a lower cross-link density and a less rigid structure on the molecular level. This less rigid structure is expressed in the higher activation energy for the mixture $(42 \mathrm{~kJ} / \mathrm{mol}$ on the basis of the phosphorescence intensity data) than for III (23 kJ/ mol on the basis of the phosphorescence intensity data).

Two remarkable observations can be made when the results obtained from the intensity data are compared with the triplet excited state half-life data. The activation energies as obtained from the intensity data are always higher than those obtained from the half-life data. The temperature independence of absorption and intersystem crossing signifies the direct relation between phosphorescence intensity and half-life. ${ }^{19}$ Hence differences in temperature dependence of both parameters can only be explained by differences in the concentration of emitting species. The same concentration effect can be found in the thermal history experiments of the polyepoxide networks. There are two reasonable explanations for this minor change in concentration of emitting species. First, there is a change in the total concentration of internal probe molecules and hence in molecules that will emit phosphorescence. Second, the total amount of probe molecules is constant, but the fraction of emitting species is changed. The second explanation is in good agreement with our observations during the formation of the networks, as published previously. ${ }^{20}$ From those experiments we were prompted to conclude that the polyepoxide networks are composed of domains with high internal mobility and domains with lower internal mobility, yielding phosphorescent sites and nonphosphorescent sites. Differences in concentration can be explained by an increase or decrease of the volume fraction of domains with high internal mobility. Since these changes in volume fraction are very small, we do not observe a non-single-exponential decay for the half-life. From this change in volume fraction, the temperature dependence of the relation of phosphorescence intensity and half-life is seen to be the result of a slow relaxation phenomenon of the polyepoxide network. A similar history effect for phosphorescence in linear polymers has been reported by Ghiggino et al. ${ }^{14}$

From the emission spectra as a function of temperature for the networks of the individual bisepoxides and mixtures thereof, it is possible to estimate the increase of molecular mobility and subgroup motion as the temperature is raised. These parameters underlie the mechanical properties of polymeric materials. Within the range of photoinitiated cationic polymerization and cross-linking of (cyclo)aliphatic bisepoxides, the differences in activation energy above the transition temperature around $260 \mathrm{~K}$ are explained in terms of differences in the molecular architecture of the polymer networks produced. With this hitherto unknown (for networks) method it is possible to predict the motion of large parts of the network, i.e., the glass transition temperature and the width of the temperature region of that glass transition. A low activation energy in the emission experiments is indicative of a small increase in motion and thus a high $T_{\mathrm{g}}$, while a high activation energy in turn indicates a $T_{\mathrm{g}}$ slightly preceding the transition temperature of $260 \mathrm{~K}$. Furthermore, it is expected that the activation energies for subgroup motion and for motions of large parts of the network are related. Thus 
a high $E_{\mathrm{a}}$ for emission indicates a $T_{\mathrm{g}}$ over a short temperature range, and a low $E_{\mathrm{a}}$ for emission indicates a $T_{\mathrm{g}}$ over a broad temperature range.

In order to determine the glass transition temperature of the polyepoxide networks and to compare these data with the estimations from the emission spectra, we performed dynamic mechanical thermal analysis at three different frequencies. When the DMTA data for the different polyepoxide networks are compared, there is only one low-temperature transition around $210 \mathrm{~K}$ that is observed in all networks, and it is probably due to the motion of a group common to all polyepoxides. In several studies of the mechanical relaxation mechanism of epoxide networks (cross-linked with amines or acid anhydrides) this low-temperature transition has often been observed, and it is suggested to be the $\beta$-relaxation due to mobile hydroxyethylene oxide end groups. ${ }^{2-5}$

The main transitions are at the glass transition temperature. A variety of temperatures and widths in temperature range are observed in our case. The main transitions are given in Table II, and the width of the temperature range in which this transition occurs is expressed in $\Delta \log E^{\prime}$ and $\Delta \log E^{\prime} / K$ values. High values for both parameters indicate a short temperature range for the glass-to-rubber transition. Most striking and remarkable is the similarity of these two parameters from the DMTA and the estimations deduced from the emission behavior of the same polyepoxides. For instance, the relatively low glass transition temperature of network made from II in combination with the short temperature range in which this transition takes place is in good agreement with the high activation energy obtained in the phosphorescence study as a function of temperature. In contrast to II, the main transition in $I$ is found at a relatively high temperature, and the transition occurs in a broad temperature range; both properties correspond to the low activation energy determined from the phosphorescence data. The frequency dependence of the mechanical transitions shows a normal behavior, to wit, a shift to lower temperature as the frequency is decreased.

The 4:6 w/w mixture of bisepoxides I and II exhibits two nonresolved main transitions. The amplitude of the tan $\delta$ of the transition at $320 \mathrm{~K}$ depends strongly on the frequency used. Going from high frequency $(10 \mathrm{~Hz})$ to low frequency $(0.1 \mathrm{~Hz})$, the amplitude of $\tan \delta$ is more than doubled. Explanations for this phenomenon are purely speculative and are not discussed here. These data indicate that at high frequencies the mixture more resembles the properties of I than those of II. This resemblance is also found in the emission experiments. Therefore we are prompted to conclude that the activation energies determined from the emission spectra correspond to molecular motion at high frequencies.

In the case of networks from monomer III the effect of purification and thus the increase in rigidity are substantial, but a good explanation is hard to give without a knowledge of the exact structure of the polar monofunctional components. The mechanical properties of the unpurified sample of III show a close similarity with the $4: 6 \mathrm{w} / \mathrm{w}$ mixture of I and II. In addition to the same position of the main transitions and the same values of $\Delta$ $\log E^{\prime}$ and $\Delta \log E^{\prime} / K$, we observed also the frequency dependence of the transition at $230 \mathrm{~K}$. This close similarity in mechanical properties is also observed in the molecular mobility and subgroup motions as determined with emission spectroscopy for III and the $4: 6 \mathrm{w} / \mathrm{w}$ mixture of I and II.

\section{Conclusions}

Throughout the years the use of luminescence from aromatic species incorporated in linear polymers has proved to be very useful in the study of molecular mobility. ${ }^{12}$ The study presented in this paper shows that polymer networks with a much more complicated structure can also be subjected to this technique. The polyepoxide networks are obtained via photoinitiated cationic polymerization of (cyclo)aliphatic bisepoxides. The intrinsic phosphorescence of this network system provides a probe for molecular motion. The data demonstrate the discontinuous behavior of phosphorescence as a function of temperature. The discontinuity is the result of the onset of quenching of the triplet excited state of the emitting species. The activation energy term governing the temperature dependence of the phosphorescence above the transition point is a direct measure of the increase of molecular motion caused by raising the temperature. These data can be explained in terms of the molecular architecture of the networks. The magnitude of the activation energy is related to the glass transition temperature and the width of the temperature range in which this glass transition occurs. This relation is clearly established by means of dynamic mechanical analysis. This study shows that a combination of techniques will provide supplementary information concerning the very complicated nature of highly cross-linked polymer networks. Although this study is limited to (cyclo)aliphatic polyepoxide networks, it is expected that emission spectroscopy and DMTA will be a useful combination in investigations into the structure and dynamics of other types of polymer networks.

Acknowledgment. We are indebted to C. J. de Boon and L. M. H. Mordang for their assistance in the experimental work, J. Boven for the synthesis of the initiators, Dr. D. M. de Leeuw for helpful discussions.

Registry No. I (homopolymer), 25086-25-3; II (homopolymer), 29611-97-0; (I)(II) (copolymer), 105336-88-7; III (homopolymer), 25085-98-7; (III)(IV) (copolymer), 105336-89-8.

\section{References and Notes}

(1) Labana, S. S., Dickie, R. A., Eds. Characterization of Highly Cross-Linked Polymers, ACS Symposium Series 243; American Chemical Society: Washington, D.C., 1984.

(2) Delatycki, O.; Shaw, J. C.; Williams, J. G. J. Polym. Sci., Polym. Phys. Ed. 1969, 7, 753 .

(3) Ochi, M.; Okazaki, M.; Shimbo, M. J. Polym. Sci., Polym. Phys. Ed. 1982, 20, 689.

(4) Shimbo, M.; Ochi, M.; Iesako, H. J. Polym. Sci., Polym. Phys. Ed. 1982, 22, 1461 .

(5) Ochi, M.; Iesako, H.; Shimbo, M. Polymer 1985, 26, 457.

(6) Cholli, A.; Ritchey, W. M.; Koenig, J. L. in ref 1, p 233.

(7) Brown, I. M.; Sandreczki, T. C. Macromolecules 1985, 18, 2702.

(8) Crivello, J. V.; Lam, J. H. W. Macromolecules 1977, 10, 1307.

(9) Crivello, J. V. Adv. Polym. Sci. 1984, 62, 1.

(10) Crivello, J. V.; Lam, J. H. W.; Volante, C. N. J. Radiat. Curing $1977,4,2$.

(11) Pappas, S. P.; Pappas, B. C.; Gatechair, L. R.; Jilek, J. H.; Schnabel, W. J. Polym. Sci., Polym. Chem. Ed. 1984, 22, 69.

(12) Somersall, A. C.; Dan, E.; Guillet, J. E. Macromolecules 1974, $7,233$.

(13) Guillet, J. E. Polymer Photophysics and Photochemistry; Cambridge University: Cambridge, U.K., 1985.

(14) Smit, J. K.; Sakurovs, R.; Ghiggino, K. P. Eur. Polym. J. 1983, 19,49 .

(15) Rutherford, H.; Soutar, I. J. Polym. Sci., Polym. Phys. Ed. $1980,18,1021$.

(16) Morawetz, H. Science (Washington, D.C.) 1979, 203, 405.

(17) Monnerie, L. In Static and Dynamic Properties of the Polymeric Solid State; Reidel: Dordrecht, The Netherlands, 1982; p 383.

(18) Tassin, J. F.; Monnerie, L. J. Polym. Sci., Polym. Phys. Ed. $1983,21,198$. 
(19) Turro, N. J. Modern Molecular Photochemistry; Benjamin/ Cummings: Menlo Park, CA, 1978.

(20) Meijer, E. W.; de Leeuw, D. M.; Greidanus, F. J. A. M.; Zwiers, R. J. M. Polym. Commun. 1985, 25, 45.

(21) Levy, R. L. Polym. Mater. Sci. Eng. 1984, 50, 124.
(22) Sung, C. S. P.; Chin, I.-J.; Yu, W.-C. Macromolecules 1985, 18 , 1510.

(23) Scarlata, S. F.; Ors, J. A. Polym. Commun. 1986, 27, 41.

(24) Yagci, Y.; Hizal, G.; Aydogan, A. C. Eur. Polym. J. 1985, 21, 25.

\title{
Perturbation Expansion of Macromolecules in Solution at the $\theta$-State
}

\section{Wolfgang Bruns}

Iwan-N.-Stranski-Institut der Technischen Universität Berlin, D-1000 Berlin 10, FRG. Received May 13, 1986

\begin{abstract}
By means of Monte Carlo methods the binary interaction terms up to the third order and the ternary interaction term of the perturbation expansion were calculated for model chains on a simple cubic lattice at the $\theta$-state (the second osmotic virial coefficient $A_{2}=0$ ) and at the state of vanishing binary interactions $\left(\beta_{2}=0\right)$. The following results were obtained: (a) theories on $A_{2}$ that are based solely on binary interaction terms fail in the vicinity of the $\theta$-state; (b) even at the $\theta$-state the configurational properties of a polymer chain cannot be described properly by a third-order perturbation theory; and (c) chains with $\beta_{2}=0$ do not exhibit pseudoideal behavior.
\end{abstract}

\section{Introduction}

The cluster expansion method of the perturbation theory was pioneered by Teramoto ${ }^{1}$ and Fixman. ${ }^{2}$ More recently this expansion has been reformulated by passing from the discrete model to a continuum limit. ${ }^{3,4}$ In each of these developments the reference system is taken to be a random-flight chain which was frequently identified with a real chain on $\theta$-point conditions. The potential of mean force between the structural units of the chain is generally described by $\delta$-function type interactions. In this case the binary cluster integral $\beta_{2}$ can be factored out and the distribution function may be expressed as a power series in $\beta_{2}$ if ternary and higher order cluster terms are ignored. Although Edwards ${ }^{5}$ could show that these expansions are not convergent but asymptotic, they were still used to study the behavior of polymer solutions in the vicinity of the $\theta$-point because the distribution of a random-flight chain is reobtained if $\beta_{2}$ vanishes. On the other hand, this is a range where the two-parameter theory fails, since terms containing the ternary cluster integral $\beta_{3}$ can no longer be neglected if $\beta_{2}$ is small. From experimental data the ternary interaction parameter is estimated to be positive near the $\theta$-point. 6 Therefore the sum of the binary cluster terms has to be negative to compensate the influence of the ternary ones.

So far the problems arising with calculations in the vicinity of the reference state have been discussed. They become even more intricate at the $\theta$-point. As has been pointed out in an earlier publication, ${ }^{7}$ the most appropriate definition of the $\theta$-state both from the theoretical and from the practical viewpoint is the vanishing of the second osmotic virial coefficient, $A_{2}$. On this basis it can be shown by comparison of various configurational properties that the $\theta$-state thus defined is not identical with a randomflight state, contradicting the statements of Flory. ${ }^{8}$ After all, it seems to be desirable to get an impression of the magnitude of the first few terms of the perturbation expansion at the $\theta$-state $\left(A_{2}=0\right)$ and at the state $\beta_{2}=0$ and to investigate to what extent configurational properties (for example, the mean square end-to-end distance $\left\langle h^{2}\right\rangle$ ) can be reproduced by a third-order theory.

\section{Basic Equations and Model}

The starting point is the perturbation expansion, which is formulated similar to that of Fixman. ${ }^{2}$ According to this theory the mean square end-to-end distance $\left\langle h^{2}\right\rangle$ is given by

$$
\begin{aligned}
& \frac{\left\langle h^{2}\right\rangle}{n l^{2}}=\frac{1-N_{1}+N_{2}-N_{3}+\ldots}{1-D_{1}+D_{2}-D_{3}+\ldots}= \\
& \ddot{1}+T_{1}-T_{2}+T_{3}-+\ldots
\end{aligned}
$$

with

$$
\begin{aligned}
N_{1} & =\frac{1}{n l^{2}} \sum_{i<j} \int \chi_{i j} r_{n}^{2} P_{0}\left(\vec{r}_{i j}, \vec{r}_{n}\right) \mathrm{d} \vec{r}_{i j} \mathrm{~d} \vec{r}_{n}=\frac{1}{n l^{2}}\left\langle\sum_{i<j} \chi_{i j} r_{n}{ }^{2}\right\rangle_{0} \\
N_{2} & =\frac{1}{n l^{2}} \sum_{i<j} \sum_{k<l} \int_{k<i} \chi_{i j} \chi_{k l} r_{n}{ }^{2} P_{0}\left(\vec{r}_{i j}, \vec{r}_{k l}, \vec{r}_{n}\right) \mathrm{d} \vec{r}_{i j} \mathrm{~d} \vec{r}_{k l} \mathrm{~d} \vec{r}_{n} \\
& =\frac{1}{n l^{2}}\left\langle\sum_{i<j} \sum_{k<l} \chi_{i j} \chi_{k l} r_{n}{ }^{2}\right\rangle_{0} \quad(i \leq k)
\end{aligned}
$$

$$
\begin{array}{r}
N_{3}=\frac{1}{n l^{2}} \sum_{i<j} \sum_{k<l} \sum_{p<q} \prime \int \chi_{i j} \chi_{k l} \chi_{p q} r_{n}{ }^{2} P_{0}\left(\vec{r}_{i j}, \vec{r}_{k l}, \vec{r}_{p q}, \vec{r}_{n}\right) \mathrm{d} \vec{r}_{i j} \mathrm{~d} \vec{r}_{k l} \\
\mathrm{~d} \vec{r}_{p q} \mathrm{~d} \vec{r}_{n}=\frac{1}{n l^{2}}\left\langle\sum_{i<j} \sum_{k<l} \sum_{p<q} \chi^{\prime} \chi_{i j} \chi_{k l} \chi_{p q} r_{n}{ }^{2}\right\rangle_{0} \quad(i \leq k \leq p)
\end{array}
$$

$$
D_{1}=\sum_{i<j} \int \chi_{i j} P_{0}\left(\vec{r}_{i j}\right) \mathrm{d} \vec{r}_{i j}=\left\langle\sum_{i<j} \chi_{i j}\right\rangle_{0}
$$

$D_{2}=\sum_{i<j} \sum_{k<l}^{\prime} \int \chi_{i j} \chi_{k l} P_{0}\left(\vec{r}_{i j}, \vec{r}_{k l}\right) \mathrm{d} \vec{r}_{i j} \mathrm{~d} \vec{r}_{k l}=$

$$
\left\langle\sum_{i<j} \sum_{k<l}^{\prime} \chi_{i j} \chi_{k l}\right\rangle_{0}
$$

$$
\begin{array}{r}
D_{3}=\sum_{i<j} \sum_{k<l} \sum_{p<q} \int^{\prime} \int \chi_{i j} \chi_{k l} \chi_{p q} P_{0}\left(\vec{r}_{i j}, \vec{r}_{k l}, \vec{r}_{p q}\right) \mathrm{d} \vec{r}_{i j} \mathrm{~d} \vec{r}_{k l} \mathrm{~d} \vec{r}_{p q}= \\
\left\langle\sum_{i<j} \sum_{k<l} \sum_{p<q} \chi_{i j} \chi_{k l} \chi_{p q}\right\rangle_{0} \quad(i \leq k \leq p)
\end{array}
$$

$P_{0}\left(\vec{r}_{i j}, \ldots, \vec{r}_{s t}\right)$ means the unperturbed multivariate distribution function of $\vec{r}_{i j}, \ldots, \vec{r}_{s t}, n$ and $l$ are the bond number 